\title{
Blood lymphocyte subsets in rats with adjuvant arthritis
}

\author{
A Franch, C Castellote, $M$ Castell
}

\begin{abstract}
Objectives-To determine the phenotype of peripheral blood lymphocytes during the time-course of adjuvant arthritis (AA) to detect alterations that could be involved in the pathogenesis of the arthritic process.
\end{abstract}

Methods-Phenotype analysis was performed on days $7,14,21,28,42,56$ and 70 after arthritis induction using monoclonal antibodies to CD5, CD4 and CD8 subsets, and flow cytometry. The proportion of activated lymphocytes and lymphocytes was also assessed with monoclonal antibodies to IL-2R (CD25), to Ia antigen and by polyclonal antibodies to rat Ig.

Results-Adjuvant arthritis produced leukocytosis with neutrophilia. Rats with AA showed a marked increase in the number of both $\mathrm{CD4}^{+}$and $\mathrm{CD8}^{+}$cells. The ratio CD4/CD8 decreased because the rise in $\mathrm{CD8}^{+}$cells was more pronounced than the increase in $\mathrm{CD4}^{+}$cells. Changes in lymphocyte counts showed two welldefined periods: the first, from day 14 to day 28 , during which the inflammation of the joints reached a maximum and changes in lymphocyte subsets were more pronounced, that is, there was a threefold increase in $\mathrm{CD8}^{+}$lymphocytes over normal counts, and the second, from day 42 to day 70 , in which modified parameters improved considerably but remained different from controls.

Conclusion-Alterations were detected in the phenotype of peripheral blood lymphocytes in AA, which provides an additional marker of disease activity.

(Ann Rheum Dis 1994; 53: 461-466)

Adjuvant arthritis (AA) is a well recognised model of rheumatoid arthritis, developed in 1956 by Pearson. ${ }^{27}$ It has been extensively used to study the pathogenesis of inflammatory arthritis and to assess the action of antiinflammatory and anti-rheumatic drugs. AA is induced in rats by injection of heat-killed mycobacteria and is characterised by a severe polyarthritis. The exact antigen that develops this experimental model is still unknown although T-cell-mediated mechanisms are involved in its pathogenesis. ${ }^{31}$ Antibodies against autologous components have been found in sera of animals with adjuvant arthritis. ${ }^{614}$ Although cell immune mechanisms are involved in AA, little is known about the quantitative relationship of lymphocyte subpopulations in adjuvant arthritic rats.

The aim of this study is to determine the phenotype of peripheral blood lymphocytes during the time-course of adjuvant arthritis to detect alterations that could be involved in the pathogenesis of the arthritic process.

\section{Materials and methods}

Female Wistar rats (Charles River, Barcelona) aged 10 weeks and weighing $180-200 \mathrm{~g}$ were housed in cages with free access to commercial pellet chow (UAR-A-04 ${ }^{\mathrm{R}}$ ) and water ad libitum. Temperature $\left(21 \pm 1^{\circ} \mathrm{C}\right)$ and light cycle (on from 8:00 to 20:00 hours) were controlled. Housing conditions were clean but not pathogen free. Animals were left for two weeks to acclimatise to the housing conditions before the experiments began.

INDUCTION AND EVALUATION OF ARTHRITIS AA was induced by a single subplantar injection $(0 \cdot 1 \mathrm{ml})$ of heat-killed Mycobacterium butyricum (Difco Laboratories, Detroit, MI) suspended in mineral oil $(5 \mathrm{mg} / \mathrm{ml})$ in the right hind-paw. A group of untreated animals matched by age and sex to arthritic animals were used as healthy controls. The severity of AA was quantified by the volume increase in the left hind-paw. On day 0 (before induction) and subsequently, $7,14,21,28,42,56$ and 70 days after adjuvant injection, the animals were weighed, and left- and right-hind-paw volumes were measured using a mercury plethysmometer (Ugo Basile, Milan). On day 14, those rats whose left-hind-paw volume increase exceeded the average left-hind-paw volume increase of the control group plus two standard deviations, were selected as arthritic rats.

\section{BLOOD SAMPLES}

At $7,14,21,28,42,56$ and 70 days after arthritis induction rats were anaesthetised with ether, and blood samples $(5 \mathrm{ml})$ were taken, by cardiac puncture, into heparin-coated sterile Venoject vacuum tubes. Blood samples were taken from 8 healthy control animals and 10 arthritic rats between 8.00 and 9.00 am.

\section{CELL COUNTS}

The number of leukocytes from each rat was determined using a counting chamber (Kova, ICL Scientific), and differential analysis of every sample was performed on staining blood smears. 
CELL SEPARATION, LABELLING AND FLOW CYTOMETRY

Rat mononuclear cells were isolated, labelled and analysed by flow cytometry as described previously. ${ }^{13}$ Briefly, rat mononuclear cells were isolated by Ficoll-Isopaque gradient centrifugation, using the method of Boyum as modified by Davidson and Parish, ${ }^{9}$ adapted to rat $\left(\mathrm{d}=1.09 \times 10^{3} \mathrm{~kg} / \mathrm{m}^{3}\right)$. Viability of each lymphoid cell suspension was assessed using acridine orange-ethidium bromide staining.

Mononuclear cells $\left(0.5-1 \times 10^{6}\right)$ were incubated with monoclonal antibodies to CD5 (OX19) as T lymphocyte marker (purchased from Sera-Lab); ${ }^{8}$ CD4 (W3/25) as T helper/ inducer marker; ${ }^{33} \mathrm{CD} 8$ (OX8) as T suppressor/ cytotoxic marker; ${ }^{4}$ Ia molecule (OX6) as marker of $\mathrm{B}$ cells and activated $\mathrm{T}$ cells; ${ }^{24}$ and CD25 (OX39) as marker of activated T cells ${ }^{26}$ (these monoclonal antibodies were kindly provided by Dr A Williams, Oxford, UK). FITC-conjugated goat $F\left(a^{\prime}\right)_{2}$ anti-mouse IgG antibodies (Sigma) were added to stain mouse monoclonal antibodies. B lymphocytes were also directly labelled with FITC-conjugated rabbit anti-rat immunoglobulins (Dako). Labelled cells were fixed in $1 \%$ paraformaldehyde in saline solution and stored in the dark at $4^{\circ} \mathrm{C}$ until analysis. All samples were analysed within 6 days of lymphocyte labelling, but determinations performed after 21 days did not reveal loss of positive fluorescence staining over time.

The percentage of fluorescent lymphocytes was determined using a FACScan flow cytometer (Becton Dickinson, Mount View, CA). Analysis gates were set in such a way that $\mathrm{T}$ or B lymphocytes were selected. ${ }^{13}$ Results for each lymphocyte subset are expressed as a percentage of positive cells compared with total lymphocytes and as the number of cells per litre of blood compared with lymphocyte count.

Statistical analysis was performed by means of an ANOVA of linear models ${ }^{19}$ considering the following causes of variation: arthritis (induced animals versus healthy control animals) and time (day of the study).

\section{Results}

HIND-PAW INFLAMMATION AND BODY WEIGHT The injected right-hind-paw volume increases in induced animals were significantly higher than control from the first day of induction (from day 7 to day 70, p<0.0001) (fig 1A). Left-hind-paw volume increases, which represent the systemic inflammation, were not detected until 10-14 days after induction. On day 14 the incidence of arthritis was 64/70. The maximum inflammation was seen on days 21 and 28. There was a slight decrease in systemic inflammation on days 42,56 and 70 , but without reaching reference values (from day 14 to day $70 ; p<0 \cdot 0001$ ). From day 14 two well-defined phases were noted: a first period or period 1 , from day 14 to 28 , during which inflammation of the joints reached a maximum, and a second phase or period 2, from day 42 to 70 , in which values decreased, although they remained higher than controls.
Animals in which arthritis had been induced also gained less weight in the first period after induction (fig 1B), which was significantly lower than controls on days 14-42 ( $<<0.005)$. In advanced phases, arthritic weight increases tended to return to reference values and body weight was restored at the end of the period studied. The evolution of both hind-paw inflammation and body weight increases showed significant differences between period 1 (days 14-28, maximum systemic inflammation) and period 2 (days $42-70$, tendency to control values) $(p<0.005)$

\section{LEUKOCYTE COUNT AND LEUKOCYTE}

DIFFERENTIAL ANALYSIS

From day 7 post-induction there was leukocytosis (fig 2A), which was more pronounced in period 1 than in period 2 $(\mathrm{p}<0.0001)$.

In relation to leukocyte differential analysis, neutrophil and lymphocyte percentages are shown in fig $2 \mathrm{~B}$. The arthritic process did not modify the percentages of eosinophils or monocytes, while basophils were virtually absent. In contrast, from day seven $A A$ induction produced an increase in neutrophil percentage (about $300 \%$ in relation to control values on days 14-28, $\mathrm{p}<0.0001)$ and a reduction in lymphocyte percentage $(30 \%$ decrease in relation to control values on days $14-28, \mathrm{p}<0.0001)$. Both types of leukocyte
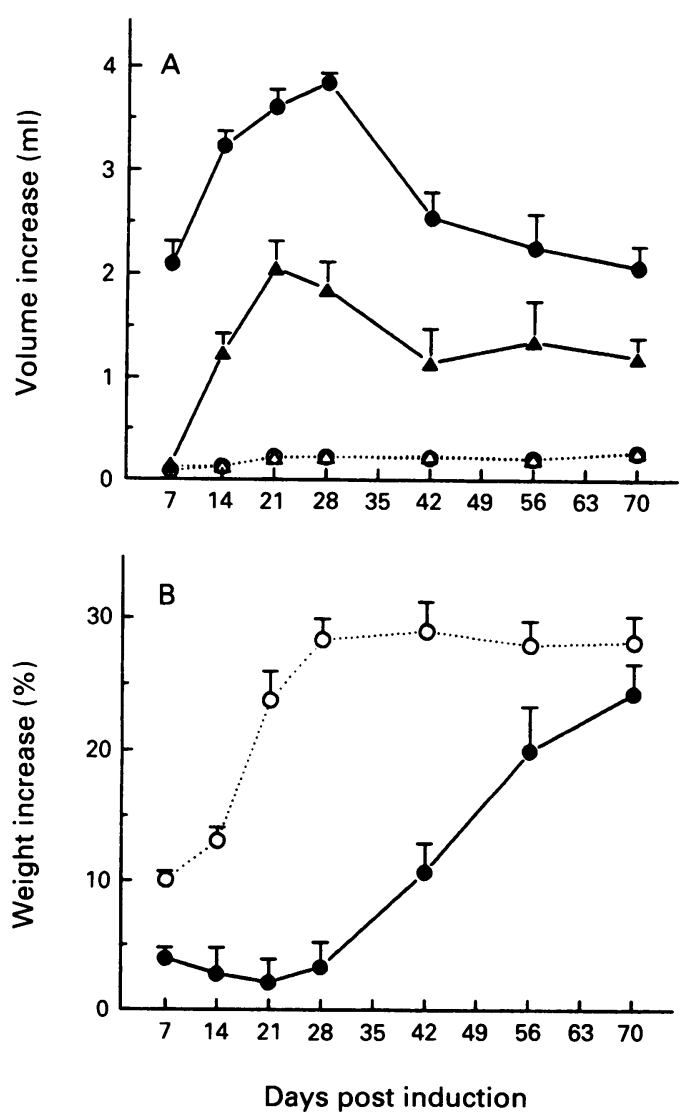

Figure 1 A) Evolution of right (circles) and left hind-paw (triangles) volume increases (ml) in arthritic (filled) and healthy (hollow) animals; B) Evolution of body weight increase in arthritic $(\bullet)$ and healthy $(0)$ animals. Each point represents the mean (SEM) of 8-10 animals. 

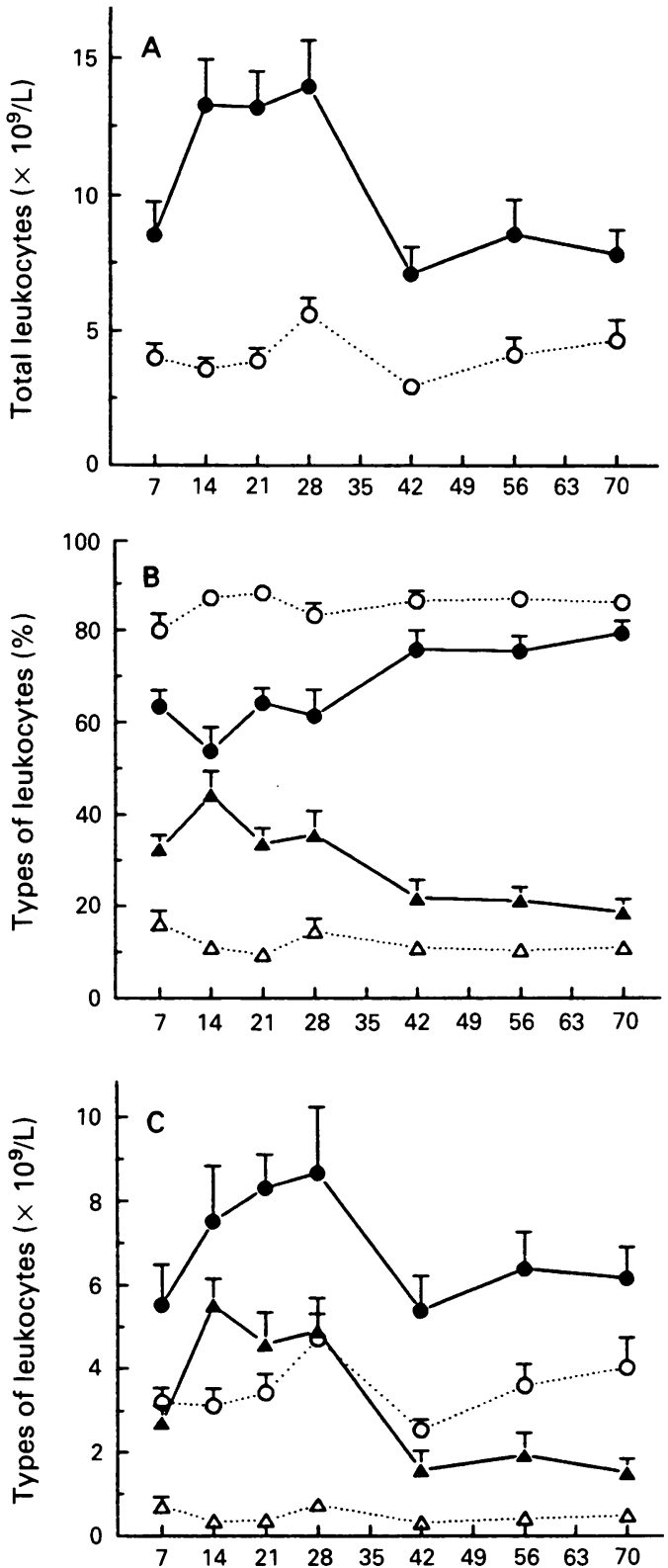

Days post induction

Figure 2 Leukocyte count and leukocyte differential analysis: A) Number of leukocytes in healthy (O) and arthritic $(\bullet)$ rats. Percentage $(B)$ and absolute number $(C)$ of lymphocytes (circles) and neutrophils (triangles) in healthy (hollow) and arthritic (filled) animals. Each point represents the mean (SEM) for 8-10 animals.

followed the two well-defined periods in the evolution of arthritis and the values in these phases were significantly different $(p<0.0001)$.

Time-courses of absolute numbers of neutrophils and lymphocytes are shown in fig 2C. Neutrophils increased significantly in arthritic animals, up to 10 fold in period 1 $(p<0.0001)$, while in period 2 the increase was only 4 fold $(p<0.01)$. Although the percentage of lymphocytes diminished in AA, their absolute number increased significantly, to double the control values, throughout the study $(p<0.05)$. There was a significant difference between the two periods of the arthritic process in the time-course of neutrophil and lymphocyte numbers $(\mathrm{p}<0 \cdot 005)$.
SUBSETS OF LYMPHOCYTES

Results of B-lymphocyte analysis are shown in fig 3. B-cells were determined in two ways: FITC-conjugated anti-Ig and anti-Ia monoclonal antibody. The results obtained with these two labels were similar, and only results for $\mathrm{Ia}^{+}$cells are reported. AA induction increased the absolute number of $B$ lymphocytes during period 1 of systemic inflammation $(p<0.005)$, but their percentage was not modified.

The absolute number of $\mathrm{CD}^{+}$cells (fig 3 ) increased throughout the study $(p<0.001)$; the increase was more pronounced in period 1 (maximum systemic inflammation) than in period 2 (slight remission of inflammation), the differences between them being significant $(\mathrm{p}<0.01)$. No difference in $\mathrm{CD}^{+}$cell percentages was observed between arthritic and control group.

The absolute number of $\mathrm{CD}^{+}$cells (fig 3) increased in the peripheral blood of adjuvant arthritic rats $(p<0.001$ compared with control values) in a biphasic pattern, following hind paw inflammation $(\mathrm{p}<0.01$, difference between periods). The percentage of $\mathrm{CD}^{+}$subset was not modified by the arthritic process.

Absolute numbers of $\mathrm{CD}^{+}$cells (fig 3 ) were considerably higher in arthritic animals during period $1(\mathrm{p}<0.0001$, compared with healthy control values); they later diminished but did not reach reference values. The percentage of $\mathrm{CD}^{+}$cells was significantly higher in arthritic rats, than in healthy animals, on day 14 postinduction $(p<0.05)$. The percentage remained high throughout the maximum inflammation period or period $1(p<0.0001)$. There was a positive correlation between the percentage of $\mathrm{CD}^{+}$cells and systemic inflammation measured in left-hind-paw $(r=0.3321$, $\mathrm{p}<0 \cdot 01)$. In the last phase studied no difference in $\mathrm{CD}^{+}$lymphocyte percentage, in relation to healthy animals, was observed. CD4/CD8 lymphocyte ratio (fig 4) was significantly lower in arthritic rats than in healthy rats on days 21 and 28 post-induction $(\mathrm{p}<0.025)$, reaching reference values in the last period.

To quantify activated lymphocytes the antiIL2R MAb was used. The percentage of $\mathrm{CD}^{2} 5^{+}$cells was always less than $2 \%$ (background value), which indicated the absence of these cells from the blood of arthritic animals.

\section{Discussion}

This study describes the time-course of phenotypes of peripheral blood lymphocytes in arthritic rat blood for 70 days after arthritis induction. Two well-defined periods can be established in the arthritic process: the first, from day 14 to day 28 post-induction is characterised by the onset and establishment of systemic inflammation and loss of body weight, and the second, from day 42 to day 70 , which corresponds to the chronic phase of the inflammation in which a tendency toward reference values was observed. Rats with AA showed a marked increase in the number of both $\mathrm{CD}^{+}$and $\mathrm{CD}^{+}$lymphocytes. The ratio $\mathrm{CD} 4 / \mathrm{CD} 8$ decreased because the rise in $\mathrm{CD}^{+}$ 

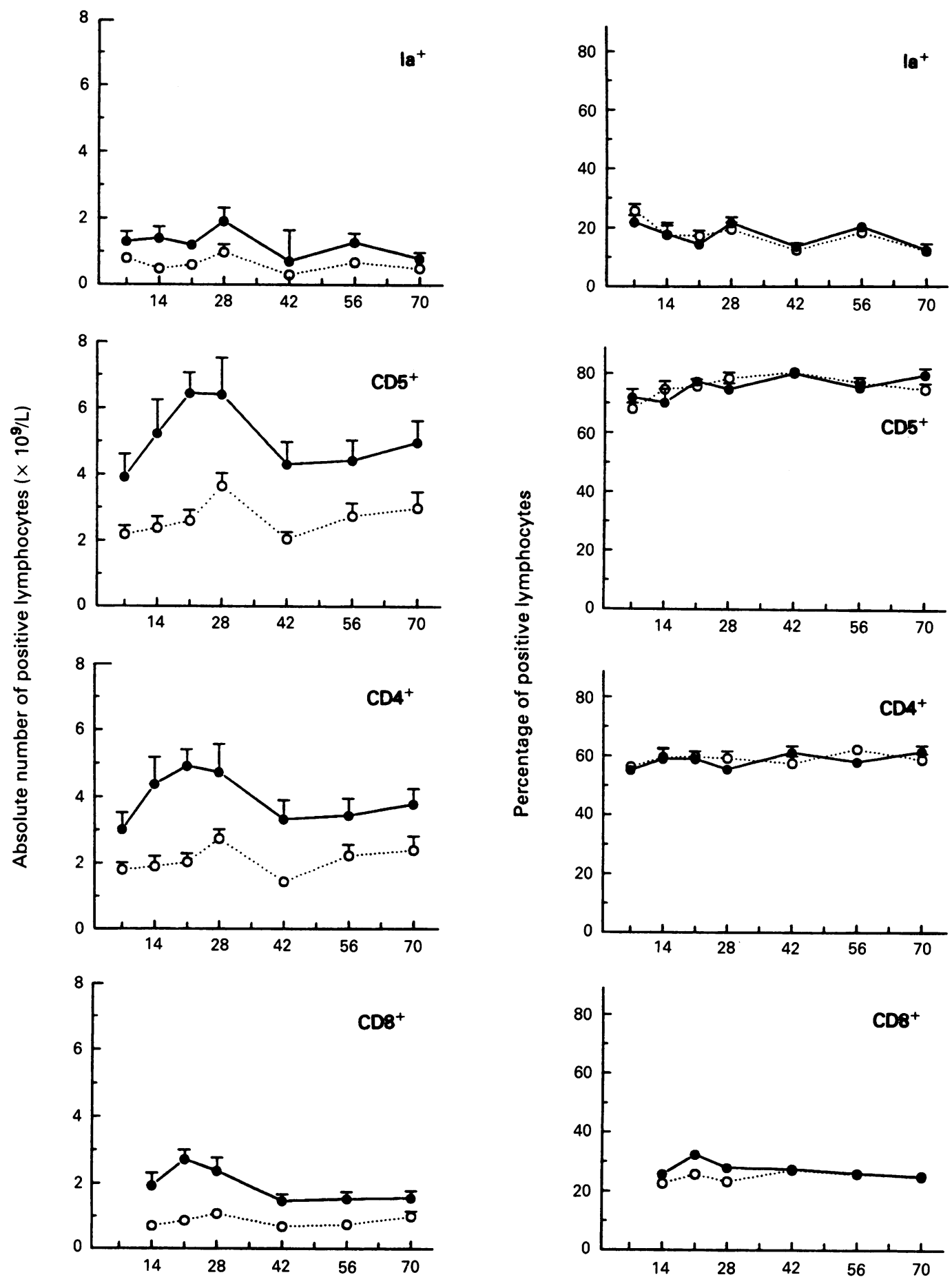

Days post induction

Days post induction

Figure 3 Absolute number and percentage of $\mathrm{Ia}^{+}, \mathrm{CDS}^{+}, \mathrm{CD}^{+}$and $\mathrm{CD} 8^{+}$lymphocytes in healthy (O) and arthritic ( $\bullet$ ) rats. Each point represents the mean (SEM) for 8-10 animals.

cells was more pronounced than the increase in $\mathrm{CD}^{+}$cells. The CD8 counts during the time-course of adjuvant arthritis followed the same pattern as the degree of inflammation measured as hind paw swelling. During the period in which inflammation of the joints reaches a maximum $\mathrm{CD}^{+}$cell counts increase threefold. However, when articular swelling diminished, $\mathrm{CD}^{+}$values improved considerably although they were still higher than controls.

Adjuvant arthritis is a well-known model of rheumatoid arthritis. However, to our knowledge, there have been very few studies on the phenotype of circulating lymphocytes in AA. The remarkable shift in phenotype that we have detected has not been reported previously, probably because it is more easily appreciated when absolute counts are considered.

The CD8 molecule is present in cytotoxic and suppressor lymphocyte subsets; thus it is not possible to attribute the percentage increase in $\mathrm{CD8}^{+}$cells to a determinate function. An increase in $\mathrm{CD}^{+}$subset has been reported by Kaufmann ${ }^{20}$ in human mycobacterial infectious diseases, and was attributed to cytotoxic activity. Although AA is 


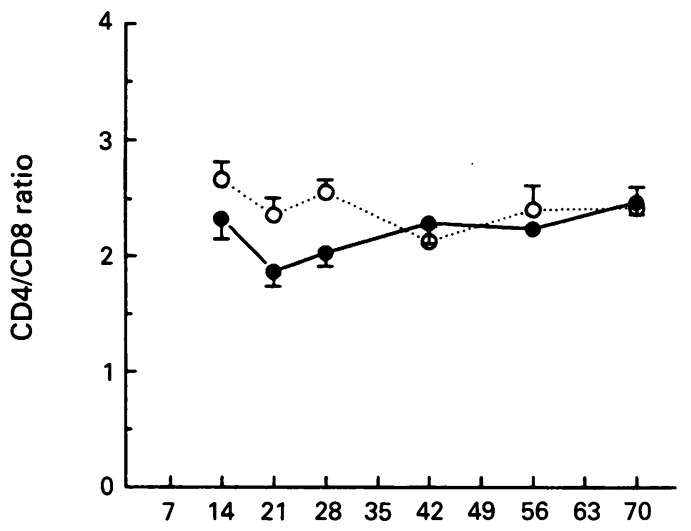

Days post induction

Figure 4 CD4/CD8 lymphocytes ratio in healthy (O) and arthritic (๑) animals. Each point represents the mean (SEM) for 8-10 animals.

induced by heat-inactivated mycobacteria, a similar mechanism could be suggested. On the other hand, the increase in $\mathrm{CD}^{+}$cells might be due to a $T$-cell bearing gamma-delta receptors, some of which are $\mathrm{CD}^{+17}$ and recognise mycobacterial determinants. ${ }^{329}$

Although alterations in the number and percentage of $\mathrm{CD}^{+}$lymphocytes in $\mathrm{AA}$ has not been described before, the role of $\mathrm{CD}^{+}$ lymphocytes in this experimental model was studied by Larsson et al, ${ }^{23}$ who observed that depletion of the $\mathrm{CD}^{+}$subset before AA induction did not modify the arthritic process. Other authors, however, ${ }^{1} 2122$ have related this subset to susceptibility and development of AA. Moreover, Binderup ${ }^{2}$ has shown that ConA-induced T-suppressor cell activity decreased in rats with $\mathrm{AA}$, and this reduction was found to coincide with the development of the polyarthritic lesions. In a recent study on arthritic rat synovial tissue we observed a marked increase in $\mathrm{CD}^{+}$cells in lining and subintimal layer, without quantitative fluctuations in $\mathrm{CD}^{+}$cells (Pelegrí et al submitted). Further, a functional study of the $\mathrm{CD}^{+}$cells, the number of which increase in $\mathrm{AA}$, is required to determine whether there is an increase in the T-cytotoxic subset (as has been described in clinical mycobacterial disease ${ }^{20}$ ) or whether there is an increase in the T-suppressor subset, but their activity is reduced.

If we compare our results with those reported for human RA, some similarities emerge. Thus the number of $\mathrm{CD}^{+}$cells has been shown to increase ${ }^{32}$ but also to remain normal. ${ }^{10}{ }^{15} \mathrm{CD}^{+}$cell number was seen to be similar to ${ }^{1012}$ or lower than ${ }^{11} 1832$ healthy reference values. Other studies do not report any difference in CD4/CD8 ratio in patients with RA. ${ }^{1025}$ Regarding blood T-lymphocyte function, contradictory results have also appeared (reviewed in reference 28 ).

On the other hand, by means of anti-Ia and anti-CD25 MAb, activated lymphocytes were not detected in arthritic rats, although they have been described in human RA. ${ }^{18} 2530$ In AA, activated $T$ cells have been reported only in arthritic synovia. ${ }^{23}$
Our total and differential leukocyte study in adjuvant arthritis also revealed leukocytosis with a high percentage of neutrophils and a high number of lymphocytes. Our data confirm and complete those of Glenn et al, ${ }^{16}$ who reported leukocytosis, neutrophilia and an increase in the number of lymphocytes on day 21 post-induction. These changes agree with results reported by Carlson et $a l^{5}$ on day 49 post-induction, who also found an increase in monocyte percentage. These fluctuations in white blood cells are probably secondary to the inflammatory process present in AA.

In summary, we have detected alterations in the phenotype of peripheral blood lymphocytes in AA and its correlation with the inflammatory process, which provides an additional marker of disease activity. The specificity of this expanded population of CD8 cells remains unknown. Studies in which peripheral blood lymphocytes and the lymphocytes infiltrating the synovium are characterised in parallel may indicate what proportion of these $\mathrm{CD}^{+}$are involved in pathogenesis.

This study was supported by a CIRIT grant, and A Franch was the holder of a fellowship from the Departament d'Ensenyament de la Generalitat de Catalunya.

We are very grateful to Dr R Pujol Borrell and Dra E Tolosa (Servei d'Immunologia de l'Hospital Universitari 'Germans Trias $i$ Pujol', Badalona) for use of flow cytometry facilities. We thank $\mathrm{Mr}$ Robin Rycroft for his valuable assistance in the preparation of the English manuscript.

1 Bersani-Amado C A, Duarte A J S, Tanji M M, Cianga M, Jancar S. Comparative study of adjuvant induced arthritis in susceptible and resistant strains of rats. III Analysis of lymphocyte subpopulations. I Rheumatol 1990; 17: 153-8.

2 Binderup L. Decreased T-suppressor cell activity in rats with adjuvant arthritis. Ann Rheum Dis 1983; 42: 693-8.

3 Born W, Hall L, Dallas A, et al. Recognition of a peptide antigen by heat shock reactive $\tau / \delta \mathrm{T}$ lymphocytes. Science 1990; 249: 67-9.

4 Brideau R J, Carter P B, McMaster W R, Mason D W, Williams A F. Two subsets of rat T lymphocytes defined with monoclonal antibodies. Eur f Immunol 1980; 10: 609-15.

5 Carlson R P, Datko L J, O'Neill-Davis L, et al. Comparison of inflammatory changes in established type II collagenand adjuvant-induced arthritis using outbred Wistar rats. Int f Immunopharmac 1985; 7: 811-26.

6 Castell M, Castellote C, Queralt J, Barberá G, Torralba A. Evidence of autoantibodies in rats with adjuvant-induced arthritis. Allergol Immunopathol 1985; 13: 399-403.

7 Castell $M$, Castellote C, Queralt J. Anti-immunoglobulin antibody detection in adjuvant arthritis by an ELISA technique. Pathol Res Pract 1986; 181: 664-7.

8 Dallman M J, Thomas M L, Green J R. MRC OX-19, a monoclonal antibody that labels rat $\mathrm{T}$ lymphocytes and augments in vitro proliferative responses. Eur $\mathcal{F}$ Immunol 1984; 14: 260-7.

9 Davidson W F, Parish C R. A procedure for removing red cells and dead cells from lymphoid cell suspensions. $\mathcal{f}$ Immunol Methods 1975; 7: 291-300.

10 Duke O, Panayi G S, Janossy G, Poulter L W, Tidman N. Analysis of $T$ cell subsets in the peripheral blood and synovial fluid of patients with rheumatoid arthritis by means of monoclonal antibodies. Ann Rheum Dis 1983; 42: 357-61.

11 Emery P, Gentry K C, Mackay I R, Muirden K D, Rowley $M$. Deficiency of the suppressor inducer subset of $T$ lymphocytes in rheumatoid arthritis. Arthritis Rheum 1987; 30: 849-56.

12 Førre $\emptyset$, Thoen J, Doblough J H, et al. Detection of T-lymphocyte subpopulations in the peripheral blood and the synovium of patients with rheumatoid arthritis and the synovium of patients with rheumatoid arthritis and
juvenile rheumatoid arthritis using monoclonal juvenile rheumatoid arthritis using
antibodies. Scand $\mathcal{F}$ Immunol 1982; 15: 221-6.

13 Franch A, Castellote C, Pelegrí C, Tolosa E, Castell M. Blood B, T, CD4 ${ }^{+}$and CD8 ${ }^{+}$lymphocytes in female Wistar rats. Ann Hematol 1993; 67: 115-8.

14 Franch A, Castellote C, Vilà J L, Vilaró S, Castell M. Anticytoskeletal autoantibody development in adjuvant arthritis. F Rheumatol, 1994; 21: 489-97.

15 Fox R I, Fong S, Sabharwal N, Carstens S A, Kung P C, Vaughan J H. Synovial fluid lymphocytes differ from peripheral blood lymphocytes in patients with rheumatoid arthritis. F Immunol 1982; 128: 351-4.

16 Glenn E M, Gray J, Kooyers W. Chemical changes in adjuvant-induced polyarthritis of rats. Am $\mathcal{F}$ Vet Res 1965 ; 26: $1195-203$. 
17 Goodman T, Lefrancois L. Expression of the $\tau-\delta$ T-cell receptor on intestinal $\mathrm{CD} 8^{+}$intraepithelial lymphocytes. Nature 1988; 333: 855-8.

18 Goto M, Miyamoto T, Nishioka K, Okumura K. Selective loss of suppressor $\mathrm{T}$ cells in rheumatoid arthritis patients: analysis of peripheral blood lymphocytes by 2-dimensional flow cytometry. F Rheumatol 1986; 13: 2-dimensi

19 Guttman I. Linear models, an introduction. New York: J Wiley and Sons, 1982.

20 Kaufmann SHE CD8 ${ }^{+} \mathrm{T}$ lymphocytes in intracellular microbial infections. Immunol Today 1988; 9: $168-74$

21 Kayashima K, Koga T, Onoue K. Role of T lymphocytes in adjuvant arthritis. I. Evidence for the regulatory function of thymus-derived cells in the induction of the disease. F Immunol 1976; 117: 1878-82.

22 Kayashima K, Koga T, Onoue K. Role of T lymphocytes in adjuvant arthritis. II. Different subpopulations of $\mathrm{T}$ lymphocytes functioning in the development of the disease. F Immunol 1978; 120: 1127-31.

23 Larsson P, Holmdahl R, Dencker L, Klareskog L. In vivo treatment with $\mathrm{W} 3 / 13$ (anti-pan $\mathrm{T}$ ) but not with OX8 (anti-suppressor/cytotoxic $\mathrm{T}$ ) monoclonal antibodies impedes the development of adjuvant arthritis in rats. impedes the development of adjuvant arthritis in rats.
Immunology 1985; 56: 383-91. 4 Mc glycoproteins in rat thymus and purification from rat spleen. Eur F Immunol 1979; 9: 426-33.
25 Nakao H, Eguchi K, Kawakami A, et al. Increment of Ta positive cells in peripheral blood from patients with rheumatoid arthritis. F Rheumatol 1989; 16: 904-10

26 Paterson D J, Jefferies W A, Green J R, et al. Antigens of activated rat $\mathrm{T}$ lymphocytes including a molecule of $50,000 \mathrm{Mr}$ detected only on CD4 positive T blasts. $\mathrm{Mol}$ Immunol 1987; 24: 1281-90.

27 Pearson $C$. Development of arthritis, periarthritis, and periostitis in rats given adjuvants. Proc Soc Exp Biol Med periostitis in

28 Petersen J. B lymphocyte function in patients with rheumatoid arthritis: impact of regulatory $T$ lymphocytes and arthrits: impact of regulatory $T$ lymphocytes and
macrophages. Laegeforeningens Forlag, Thesis, Københavns macrophages. Laegeforeningens
Universitet, Denmark, 1987.

29 Raulet D H. Antigens for $\tau / \delta$ T cells. Nature 1989; 339: 342-3.

30 Russell A S. Activated lymphocytes in the peripheral blood of patients with rheumatoid arthritis. $\mathcal{F}$ Rheumatol 1990; 17: 589-96.

31 Taurog J D, Argentieri D C, McReynolds R A. Adjuvant arthritis. Methods Enzymol 1988; 162: 339-55.

32 Veys E M, Hermann P, Schindler J, et al. Evaluation of T cell subsets with monoclonal antibodies in patients with rheumatoid arthritis. $\mathcal{7}$ Rheumatol 1982; 9: 25-9.

33 White R A H, Mason D W, Williams A F, Galfre G, Milstein C. T-lymphocyte heterogeneity in the rat: separation of functional subpopulations using a monoclonal antibody. f Exp Med 1978; 148: 664-73. 